\title{
A Web System for Choose Your Own Adventure
}

\author{
Zizhong J. Wang1,2, Regina L. Barner² \\ ${ }^{1}$ College of Information Engineering, Taiyuan University of Technology, Taiyuan, China \\ ${ }^{2}$ Department of Mathematics and Computer Science, Virginia Wesleyan College, Norfolk, USA \\ Email: zwang@vwc.edu
}

Received December 2013

\begin{abstract}
The Web system for Choose Your Own Adventure (CYOA) project utilizes the PHP and MySQL programming languages for an entertainment purpose. This project creates a fully-functional and secure online user system, in which the accounts of the user system are stored in a MySQL database. Over the duration of this project, it is found that the PHP and MySQL programming languages are able to be employed for entertainment purposes, and they are both very effective in the field of entertainment. This project is meant to broaden the horizons of aspiring programmers in order to give them a glimpse into a possibility of using these programming languages in a scope that is commonly broached by game developers, but not programmers in the usual case.
\end{abstract}

\section{Keywords}

Web System; PHP/MySQL; Entertainment; Game Developer

\section{Introduction}

The online information systems or Web systems have been developed so fast in the past decades [1,2]. The importance of this project is to give programmers, both beginner and experienced, an insight into the possibilities of using the PHP and MySQL programming languages for entertainment purposes. It also allows users to broaden their horizons by delving into an interactive online text adventure that they may not have experienced before.

The goal of this project is to give a convincing example of how the PHP and MySQL programming languages could be used for entertainment purposes [3], instead of strictly analytical applications. The most effective presentation of this goal is to create an online tool that allowed users to create their own accounts and experience a text adventure of their choosing. The display properties of this project are accomplished by building web pages using extensive PHP, and to store user account information into a MySQL database which the website is able to access in order to add information [4], change information, or remove information. In the next section, the system design is presented. The implementation of the system is introduced in the Section 3. The conclusion is given in the last section. 


\section{System Design}

URL:zeus.vwc.edu/ rlbarner/cyoaabstract.html presents the system home page. The information stored in the following table, CYOAUsers, is comprised of user accounts and information pertaining to the user, such as username, password, and more. Figure 1 shows the descriptive summary of the table CYOAUsers, and Figure 2 shows the flowchart of the system.

When viewing the Website, everyone starts at the front page, which prompts the user to either register for a user account, enter the security question and answer combination if the user forgets the username and/or password, or log into the system with a preexisting user account. The front page is as Figure 3.

If the user chooses to register for a user account, he/she would be prompted to enter the name, gender, desired username, password (which is case sensitive), the chosen security question and security answer. The name and disclosed gender is general information. The username and password is used to log into the online tool. The security question and answer are used in case the user forgets either the username or password. The registration page is as Figure 4.

If the user chooses to log into the account in order to access the online tool, he/she is prompted to enter the username and password, along with a gentle reminder that both are case sensitive. The login page is as Figure 5.

After successful login, the user would arrive at the user hub, which allows the user to either choose a text adventure to pursue, or to change something in their user options, which consisted of changing the password, seeing the statistics, resetting the statistics, or removing the account. The user hub page is as Figure 6.

The "Change Your Password" form features three input boxes, which prompts the users to enter their current



Figure 1. The description of CYOAUsers table.

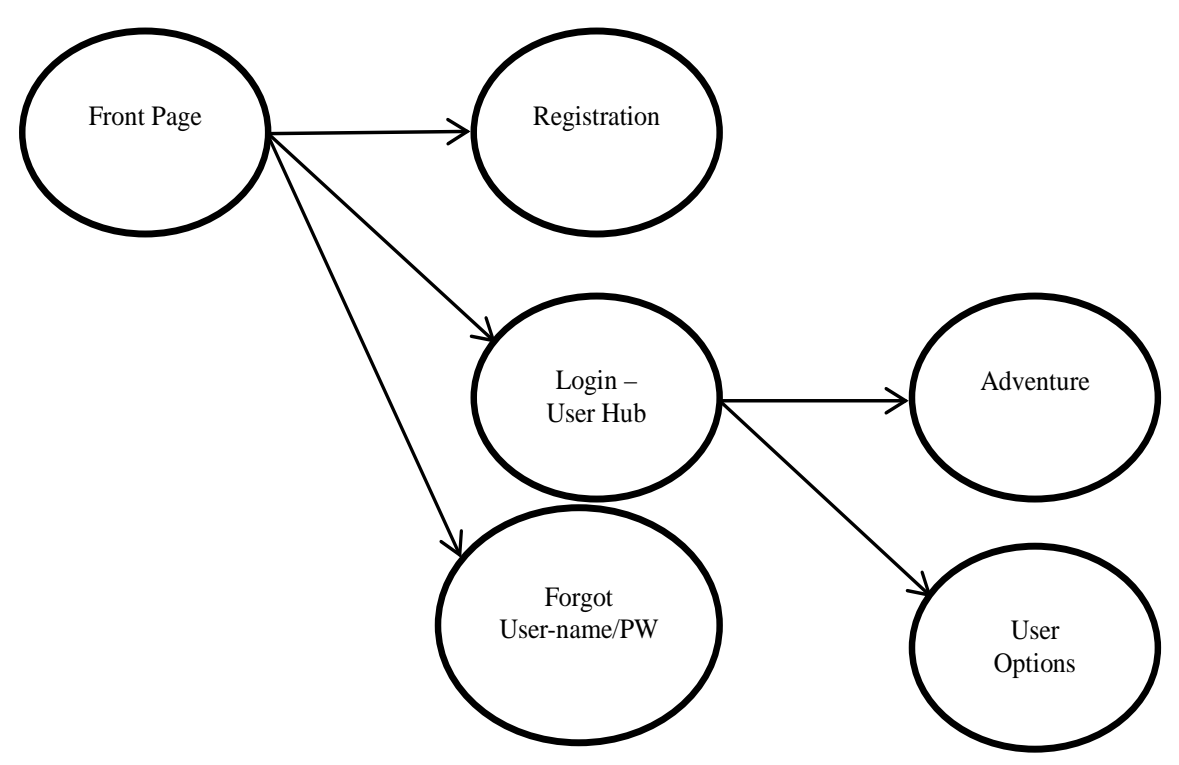

Figure 2. System flowchart. 


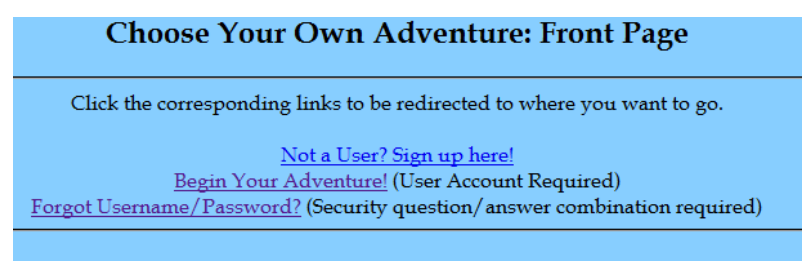

Figure 3. The snapshot of the front page of the system.



Figure 4. The snapshot of the user registration page.

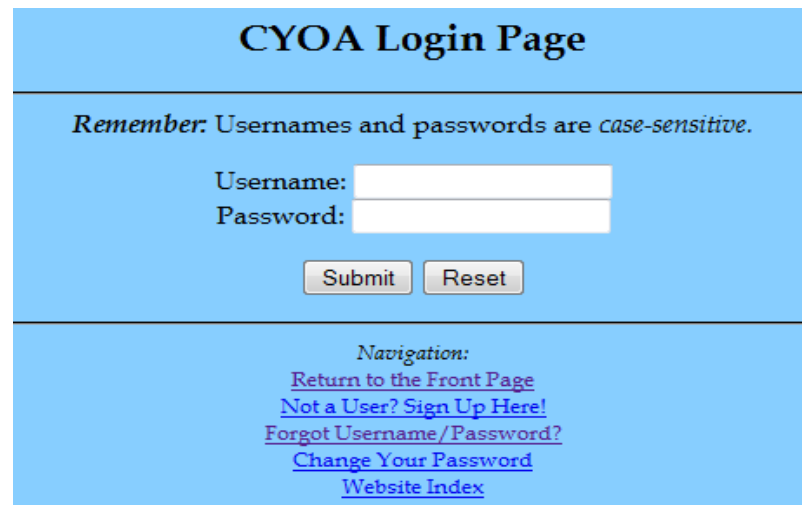

Figure 5. The snapshot of the user login page.

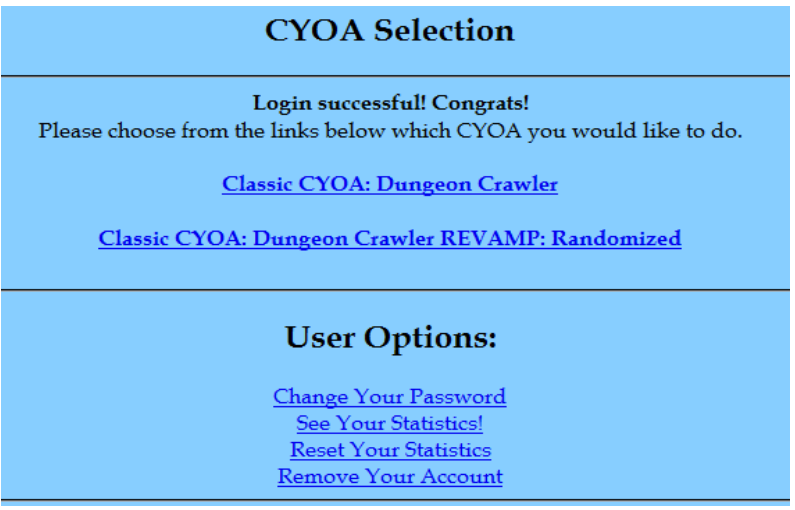

Figure 6. The snapshot of the user selection page. 
password, the new password that they wish to use, and re-input the new password. If the new password is the same as the current password used, an error message would occur and redirect the user back to the input form. If the new password and re-entered password did not match, an error message would occur and redirect the user back to the input form. The "Change Your Password" form is as Figure 7.

The "See Your Statistics" form uses session variables that stored the user's username and password in order to access the MySQL table and to display a chart that shows the user's CYOAs Started (incremented every time the user accessed a starting text adventure page), CYOAs Finished (incremented every time the user accessed a finishing text adventure page), and CYOA Deaths (incremented every time the user accessed a text adventure death page). The "See Your Statistics" form is as Figure 8.

The "Reset Your Statistics" form also uses session variables that store the user's username and password in order to access the MySQL table and to change all of the statistics, as mentioned above, back to zero if the user wishes. It would then display a chart that showed the new zero statistics, to show the user that the statistics are reset. The "Reset Your Statistics" form is as Figure 9.

The "Remove Your Account" page would use session variables that store the user's username and password in order to delete the information from the MySQL table. It would delete the account upon clicking on the link, so it is advised not to click that link unless users are sure that they wanted to delete their account. The "Remove Your Account” page is as Figure 10.

\section{System Implementation}

The Web system is implemented with over 20 HTML/PHP files. Some of the selecting pages are as follows.

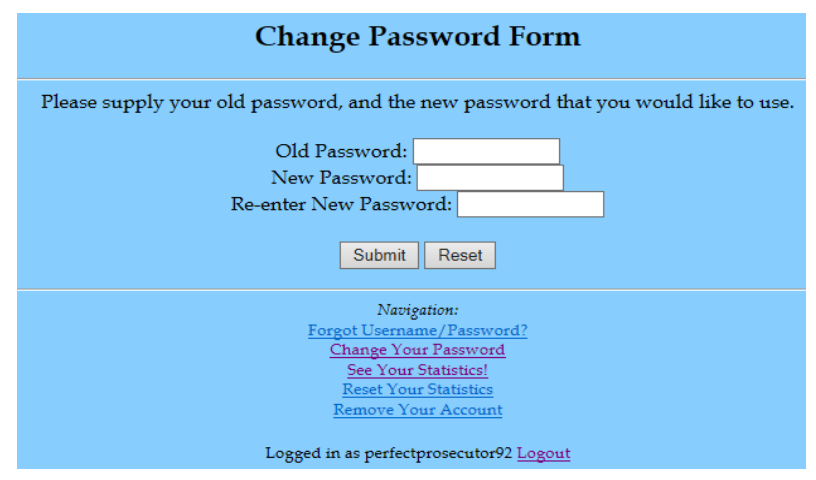

Figure 7. The snapshot of the password change page.

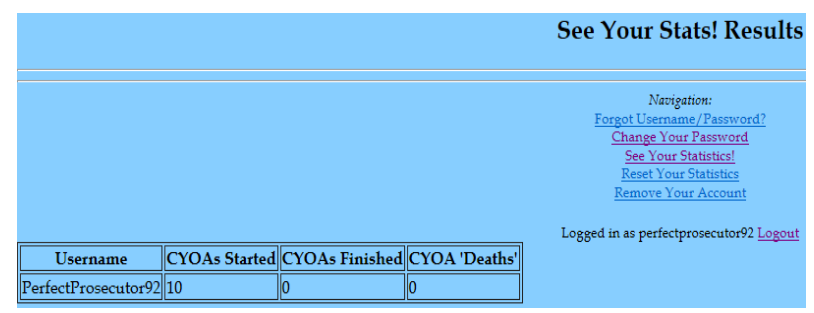

Figure 8. The snapshot of the statistics form page.



Figure 9. The snapshot of the statistics reset form page. 


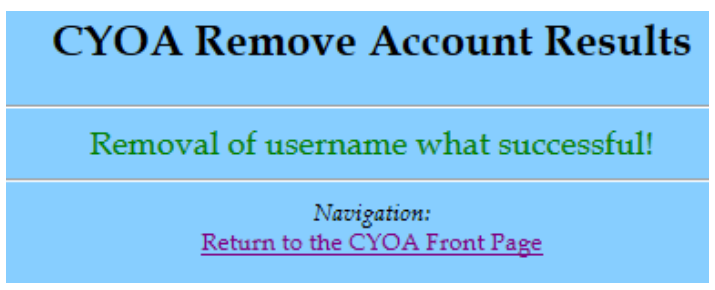

Figure 10. The snapshot of the account removal page.

- cyoafrontpage.php - The front page of the Web system that allows the user to either register for a user account, login with the user account, or to poll the MySQL table for the username and/or password if they forgot it.

- cyoaregistration.html-The new user registration page, which asks for the user's name, gender, desired username, password, security question (chosen from a drop-down list), and security answer.

- registrationaction.php - The registration results page, which displays the information that the user just inputted for their new user account [8]. If an error occurred, the user has to repeat the registration process, and no new user account would have been added to the MySQL table, CYOAUsers.

- forgotuserpass.html - If a user forgets his/her username and/or password, he/she could poll the MySQL table for their information by picking their security answer from a selection box and typing in their security answer, which is case sensitive.

- forgotuserpassactionnotin.php-The forgot username/password results page, if the user has not logged into the online tool. This page displays the user's username and password combination in a small table if their security question and answer inputs are both correct. If an error occurred, the user would have had to start the process of obtaining their information over again.

- cyoalogin.html-The main login page, which prompts the user to input their username and password. There are hidden variables embedded in the login form on this page that pertained to the "randomized" text adventure; specifically, those variables are "fire", "water", and "earth".

- cyoaloginaction.php - The user would never see this page. It consists of a java script that directed the user to cyoaloginactionb.php, after polling the MySQL table to check that their username and password combination are correct. The login session began on this page.

- cyoaloginactionb.php - The user hub, which allows the user to either choose a text adventure to pursue, change their options, or logout.

- classicprimer.php - The beginning prompt of the first text adventure, which is labeled as "Classic CYOA: Dungeon Crawler" on the website. Every page, linked to this page by a series of redirections via form buttons, involves the text adventure. Each page features a short prompt and two choices, preceded by buttons, which allowed the user to choose what they would like to pursue during the text adventure. These pages are used for the entertainment side of the project only, used as a vessel to fuel the story contained within the text adventure.

- randomstartdirect.php—Began the second of the two text adventures available on the website, titled "Classic CYOA: Dungeon Crawler REVAMP: Randomized”. Because this particular text adventure consists of four "rooms" that the user cycles through at one point or another, this page randomly chooses one of the rooms to start the user off inside, which allows for more varied text adventure experiences [6]. It is unlikely for the user to begin in the same room, even if the user began this same text adventure one play through after another. The following pages correspond to the pages linked together to form this particular randomized text adventure.

- waterlanding.php, firelanding.php, earthlanding.php, finallanding.php—“Landing” pages that increment the session variable dependent upon the element of the room. Session variables "water", "fire", and "earth" are used to track which rooms the user had previously visited. If all session variables held a "1", the user would be directed to the "finallanding.php", which led to the end of the text adventure.

- "Fire" rooms, which features a prompt and two choices preceded by a form button for redirection to other pages, includingfirebackaway.php, firefeelaround.php, firegrabjewel.php, fireputout.php, firereachinto.php, firesearch.php, firewithdraw.php. These pages are used for the entertainment side of the project only, used as a vessel to fuel the story contained within the text adventure. 
- "Water" rooms, which features a prompt and two choices preceded by a form button for redirection to other pages, includingwaterdive.php, watergetjewel.php, waterhallwaynojewel.php, waterhop1.php, waterhop2.php, waterhop3.php, waterquestion2.php, waterquestion3.php, waterwrong.php. These pages are used for the entertainment side of the project only, used as a vessel to fuel the story contained within the text adventure [7].

- "Earth" rooms, which features a prompt and two choices proceeded by a form button for redirection to other pages, included earthheart.php, earthkeyhole.php, earthturnback.php, earthusekey.php, earthxbone.php. These pages are used for the entertainment side of the project only, used as a vessel to fuel the story contained within the text adventure.

- The "final" room, which features a prompt and three radio buttons (labeled "rock", "paper", and "scissors", respectively) in order to simulate a rock-paper-scissors game against the "boss" of the text adventure, including finaldie.php, finaltie.php, finalaction.php, finalwin.php. These pages are used for the entertainment side of the project only, used as a vessel to fuel the story contained within the text adventure.

- fireredirect.php, waterredirect.php, earthredirect.php - After the user completes one of the elemental rooms, he/she would reach this page, which checks the elemental session variables to see which held "1"s. It randomly picks a room for the user to explore next, dependent upon which elemental variables held " 1 "s or " 0 "s. If all session variables contain " 1 "s, the user would be redirected to the finallanding.php page to complete the text adventure [8].

\section{Conclusion}

The Web system For Choose Your Own Adventure project explores the possibilities of using programming languages such as PHP and MySQL for entertainment purposes, instead of analytical or mathematical purposes. This project is conducted online, and utilized a MySQL database to store user account information entered and modified from the website. The importance of this project is that it could broaden the horizons and perspectives of both aspiring and experienced programmers: instead of using these languages for just an analytical or mathematical purpose, they could also be used for entertainment purposes. It is concluded that using analytical and mathematical programming languages such as PHP and MySQL for an entertainment purpose is entirely possible, and these languages are very effective being used for this purpose.

\section{References}

[1] Wang, Z., et al. (2013) The Dynamic Business Web Template Design and Implementation. Proceedings of the 12th International Conference on E-Learning, E-Business, Enterprise Information Systems, \& E-Government (EEE'13), CSREA Press, 225-228.

[2] Johnson, G. and Wang, Z. (2013) An Online Database System for Card Stores. Proceedings of the 12th International Conference on E-Learning, E-Business, Enterprise Information Systems, \& E-Government (EEE'13), CSREA Press, 229-233.

[3] W3Schools (2013) SQL UPDATE Statement. http://www.w3schools.com/sql/sql_update.asp

[4] W3Schools (2013) SQL ALTER Statement. http://www.w3schools.com/sql/sql alter.asp

[5] Javascript Message Box with "Yes" and "No" Button. http://www.webdeveloper.com/forum/showthread.php?96282-RESOLVED-Javascript-message-box-with-quot-Yes-qu ot-and-quot-No-quot-button

[6] (2013) Rand. PHP Manual. http://us2.php.net/manual/en/function.rand.php

[7] User-Defined Functions. PHP Manual. http://php.net/manual/en/functions.user-defined.php

[8] 13.7.5.5. SHOW COLUMNS Syntax. MySQL. http://dev.mysql.com/doc/refman/5.0/en/show-columns.html 